\title{
Evaluación espaciotemporal del cambio global en el Departamento Sur de Haití, de 1973 al 2017
}

Evaluation of the global spatiotemporal change in the South Department of Haiti, from 1973 to 2017

\author{
Marco A. Martínez ${ }^{1}$ (D) Christian Birkel ${ }^{1}$ Édgar Espinoza $^{1}$ (D), Melvin Lizano ${ }^{1}$, Edes Philippe $^{2}$, \\ Laura Benegas ${ }^{2}$ \\ 1 Escuela de Geografía y Observatorio del Agua y Cambio Global, Universidad de Costa Rica. \\ 2 Centro Agronómico Tropical de Investigación y Enseñanza; WATERCLIMA-LAC, Gestión y zonas costeras, Costa Rica. \\ Autor para correspondencia: marco.martinezmartinez@ucr.ac.cr \\ Fecha de recepción: 4 de junio de 2019 - Fecha de aceptación: 11 de septiembre de 2019
}

\section{RESUMEN}

Haití es una nación con grandes retos ambientales y sociales. La alta deforestación, falta de planificación territorial, inestabilidad política y económica, y una población ejerciendo presión sobre recursos naturales son factores característicos de Haití que se manifiestan en su dinámica territorial. En este artículo analizamos los procesos de cambio de cobertura de la tierra en el Departamento Sur de Haití de 1973 al 2017 y la relación de estos procesos con ciertas variables socioeconómicas y biofísicas asociadas al cambio global y sus componentes. Para ello utilizamos imágenes satelitales Landsat, repositorios de datos públicos, metodologías para el análisis de la direccionalidad del cambio, así como análisis estadísticos correlacionales. Los resultados muestran una fluctuación marcada en el cambio de áreas de cultivos, una relación parcial del suelo desnudo con zonas deforestadas, así como una disminución en la cobertura boscosa tras una serie de eventos hidrometeorológicos y sociopolíticos presentados en Haití. Además, encontramos que el cambio de matorral se asoció significativamente con las tasas de cambio de población, de temperatura y del Índice de Desarrollo Humano (IDH), mientras que la tasa de cambio de precipitación influyó significativamente en el cambio de la cobertura de cultivos. Por otra parte, los cambios en el suelo desnudo tuvieron relación significativa con la tasa de cambio de población. Finalmente, ninguna de las variables consideradas se asoció significativamente con el cambio espaciotemporal de las coberturas urbano y bosque.

Palabras clave: Cambio Global, Cambio de cobertura de la tierra, Teledetección, Haití.

\begin{abstract}
Haiti as a country faces great environmental and societal challenges. Haiti is characterized by a high deforestation, lack of territorial planning, political and economic instability, and a growing population exerting pressure on natural resources. In this article, we analyze the historic land cover change in the Southern Department of Haiti from 1973 to 2017 and the relationship to socioeconomic and biophysical variables. All variables under change together are considered as the holistic definition of global change. We used Landsat satellite images, public data repositories and statistical correlations to analyze the directionality of the above-mentioned global change in Haiti. The results showed a marked fluctuation of crop areas, a relation of bare soil with deforested areas, as well as a decrease in forest cover after a series of hydrometeorological and sociopolitical events. In addition, we found that changes in brush area were significantly associated with the rates of change in population, temperature and the Human Development Index (HDI), while the rate of change in rainfall significantly influenced the change in crop coverage. On the other hand, changes in bare soil had a significant relationship with the rate of population change. Finally, none of the variables considered was significantly correlated with the spatiotemporal change of urban and forest coverage.
\end{abstract}

Keywords: Global Change, Land cover change, Remote Sensing, Haiti.

\section{INTRODUCCIÓN}

El cambio de uso y cobertura de la tierra en el Planeta representa uno de los mayores contribuyentes a los procesos de cambio ambiental global (Geist \& Lambin, 2001; Lambin, Geist, \& Lepers, 2003; Duarte et al., 2006;
Lambin \& Geist, 2006). Estas dinámicas de cambio responden en gran parte a las crecientes presiones antropogénicas por el aumento poblacional y demanda de recursos. No obstante, diversos científicos sostienen que el cambio de uso y cobertura de la tierra está diversamente conducido, principalmente, por el sistema económico 
imperante, dinámicas demográficas locales y globales, el sistema político, el desarrollo tecnológico acelerado, modos y estilos de vida social, alteraciones climáticas y biofísicas del Planeta y la dinámica del uso del suelo (Geist \& Lambin, 2001; Lambin et al., 2001; Veldkamp \& Lambin, 2001; Carr, 2004; Zondag \& Borsboom, 2009; Goldewijk \& Ramankutty, 2010; Lambin \& Meyfroidt, 2011). Los cambios no ocurren de forma homogénea y suceden a largo plazo. Estos factores conductores del cambio pueden dividirse en muchas fuerzas impulsoras específicas que afectan elementos puntuales del sistema terrestre (Zondag \& Borsboom, 2009).

Según datos del Departamento de Asuntos Económicos y Sociales (DAES) de la ONU (2017), el Planeta Tierra en el año 2017 albergaba a 7.750 millones de personas, estimando para el 2050 más de 9.700 habitantes. Para la región de Latinoamérica y Caribe, para el 2017 había 646 millones de personas (DAES, 2017), con una proyección de 780 millones para el 2050. Por tanto, todo indica que el aumento poblacional a nivel mundial continuará ejerciendo presión sobre los recursos naturales y con ella la dinámica territorial, especialmente en los trópicos, con consecuencias a nivel global (Houghton, 1994).

Haití es país con retos importantes de esta índole, incluido el aumento poblacional, presión sobre escasos recursos naturales, alta deforestación, inestabilidad sociopolítica y económica, desastres socio-naturales, entre otros, que a su vez crean realimentaciones que agravan más la condición de vulnerabilidad socioeconómica y ambiental del país (González \& Manero, 2011). Aunado a esto, ha habido un deficiente manejo político de las autoridades del Gobierno ante estos importantes desafíos (PNUMA: Programa de las Naciones Unidas para el Ambiente, 2013). Por ejemplo, los sistemas de gobierno de Haití han incidido en los últimos siglos en una disminución considerable de su cobertura forestal, proceso que inició desde la época colonial y actualmente no se vislumbra un compromiso estatal por fortalecer y crear políticas públicas ambientales. En el siglo XV, el 85\% del territorio haitiano estaba cubierto de bosques (Barthelemy \& Barthelemy, 2003); hoy en día la cubierta forestal comprende entre el 2 y el $4 \%$ del territorio (PNUMA, 2013), en gran medida debido a la sobreutilización del recurso maderero para extraer carbón para uso doméstico y energético. Del mismo modo, no debe obviarse el aumento poblacional que Haití presenta (1.6\% de crecimiento anual), alcanzando para el año 2015 unos 10,711,067 habitantes (Banco Mundial, 2017) en tan sólo 27,750 km² $(386$ habitantes $/ \mathrm{km}^{2}$ ).

Lo anterior lleva a una reflexión sobre los posibles factores ambientales, socioeconómicos y políticos que inciden en estos cambios en el paisaje haitiano, particularmente en el Departamento Sur de Haití. Por eso, en este estudio quisimos analizar el cambio global y sus componentes, cuantificando los cambios, examinando su direccionalidad, y explorando su interrelación con ciertas variables biofísicas y sociales en este sitio de estudio. Aquí conceptualizamos el cambio global como cambios acumulativos en componentes biofísicos por acciones antropogénicas y naturales que se dan a nivel local/regional pero que tienen alcance global, por la naturaleza sistémica del funcionamiento terrestre (Meyer \& Turner, 2002). Esta interpretación del cambio global, entonces, lleva implícita las interacciones de las actividades antropogénicas y de procesos naturales, las cuales confieren una gama amplia de cambios en las condiciones físicas de la Tierra (Rounsevell, 2006; Equihua Zamora, Hernández Huerta, Pérez Maqueo, Benítez Badillo, \& Ibañez Bernal, 2015).

\section{MATERIALES Y MÉTODOS}

\section{1. Área de estudio}

El Departamento Sur de Haití (DS) está ubicado al suroeste de Haití y comprende un área de aproximadamente $2,600 \mathrm{~km}^{2}$ (Fig. 1). Cuenta con una población de 774,976 habitantes, representando poco más del 7\% del total de población del país.

El DS es catalogado como de alta vulnerabilidad social y ambiental, y está enmarcado en un sistema político inestable (González \& Manero, 2011). Entre las actividades económicas principales destacan la agricultura básica, ganadería y mano de obra para servicios. El clima es tropical, con dos épocas lluviosas, una de abril a junio, y otra de octubre a noviembre. El DS registra una pluviosidad anual promedio de $1703 \mathrm{~mm}$ y una temperatura anual promedio de $27^{\circ} \mathrm{C}$ (Administración Nacional Oceánica y Atmosférica, 2017). Gran parte de la lluvia entre julio y octubre se debe al aporte de humedad de los ciclones tropicales y huracanes que se originan en el Océano Atlántico y Mar Caribe. Además, Haití experimenta épocas interanuales donde eventos hidrometeorológicos son influyentes en la dinámica natural interna del país.

Al igual que la mayoría del país, esta zona está caracterizada por una alta deforestación y daño ambiental, siendo la erosión y la baja fertilidad de los suelos problemas determinantes (WaterClima-LAC, 2017). Al noroeste de Les Cayes, en el DS, se encuentra el 68\% del total del área del Parque Nacional Pic Macaya (Fig. 1), sitio importante para la población haitiana por conservar los pocos remanentes de bosque primario y especies de flora y fauna endémicas de La Española. Sin embargo, se estima que hay más de 53,000 personas habitando dentro de la jurisdicción del parque (Ministère de L'environnement, 2015), lo que incide en la deforestación del área protegida.

\subsection{Procesamiento de imágenes satelitales}

Como primera etapa de esta investigación, se llevó a cabo una clasificación supervisada para obtener mapas de cobertura de la tierra del DS. Para ello se utilizaron imágenes Landsat (dos "bloques" para cubrir toda el área de estudio) obtenidas del repositorio web de USGSNASA, en el rango anual 1973-2017. Al final, los años de análisis fueron 1973, 1979, 1986, 1992, 2000, 2007, 2013 y 2017, tomando como criterios de selección la cobertura nubosa menor al $10 \%$, una disponibilidad temporal de ambos bloques lo más consecutivo posible (idealmente anual o quinquenal), así como la ausencia de alteraciones radiométricas ni de radiancia en píxeles.

El preprocesamiento de las imágenes satelitales se efectuó con el software de código abierto QGis versión 2.18 Las Palmas, bajo la aplicación Semi Automatic Classification (SCP). Como primer paso en el preprocesamiento, se efectuó la corrección atmosférica para eliminar píxeles 


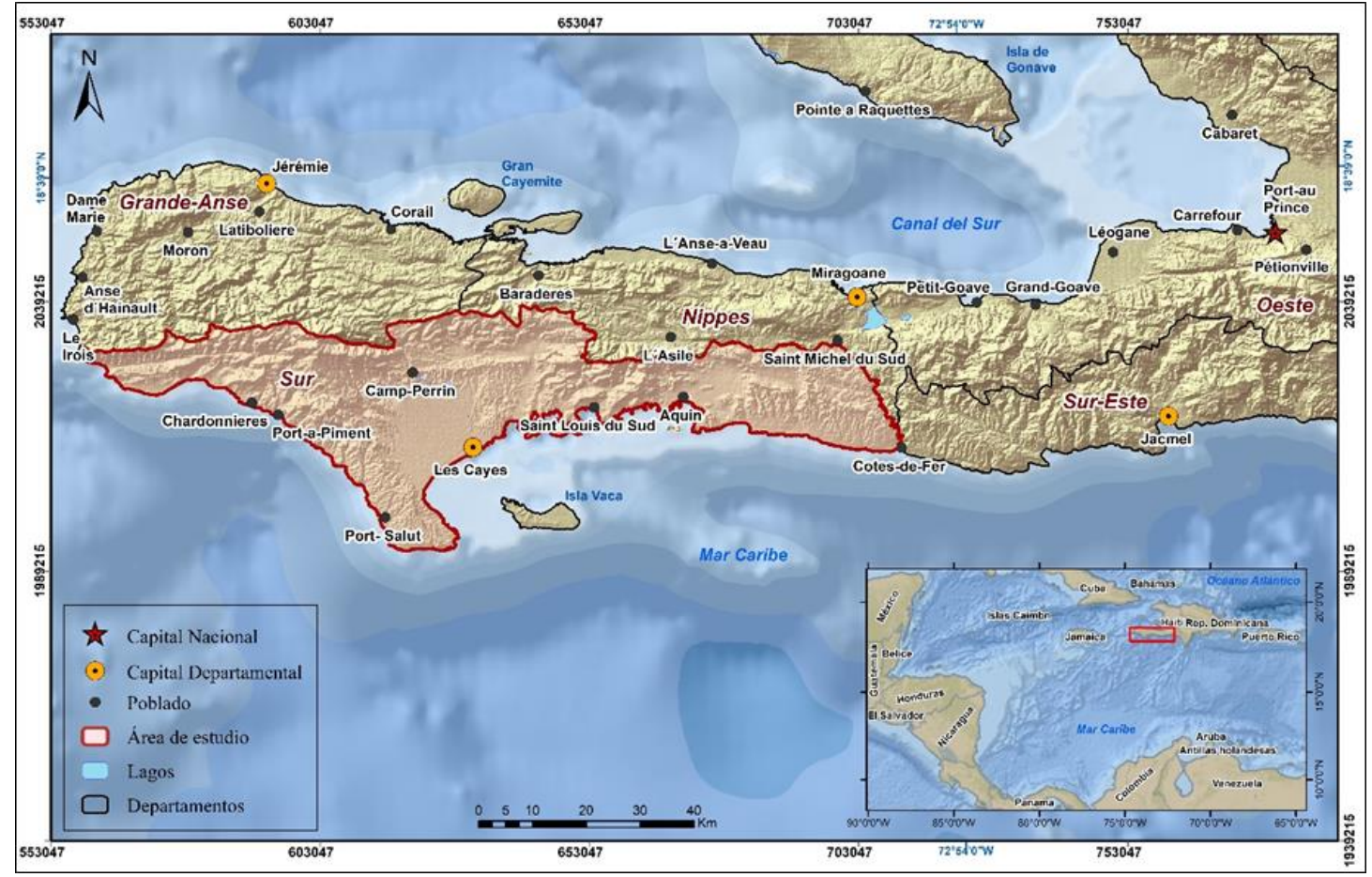

Figura 1. Zona de ubicación del estudio enmarcado en rojo en un contexto regional (cuadro inferior).

oscuros con el método DOS-1 (Chávez, 1988); luego, se realizó el juego de bandas de color verdadero (para los proyectos Landsat $4,5,7$ y 8) y falso color (para los proyectos Landsat 1, 2 y 3); finalmente, se creó el mosaico de bloques de imágenes satelitales que cubren el área de estudio. Al final, mediante el software Geomática y su herramienta "Smart GeoFill" se logró reducir la nubosidad en las imágenes satelitales a un porcentaje promedio de $10 \%$. Para esto, se debió contar con al menos otro bloque de imágenes satelitales para todos los años en estudio.

Posterior al preprocesamiento, se realizó una clasificación no supervisada del DS con el algoritmo K-means, con las siguientes clases de cobertura: cuerpos de agua, urbano o infraestructura, cultivos, bosque, pastos arbolados y suelo desnudo. Para verificación de la clasificación final, se comprobaron y reconocieron en campo aquellas zonas en las no había seguridad de existencia de una determinada cobertura, con el fin de obtener una clasificación supervisada basada en el algoritmo de Máxima Verosimilitud para todos los años en estudio. Ambas clasificaciones se realizaron con el software ERDAS IMAGINE 2015.

\subsection{Cuantificación del cambio de cobertura y determinación de la direccionalidad de cambio}

Los mapas producto de la clasificación supervisada fueron luego utilizados para calcular las tasas de cambio desde el año 1973 al 2017. Para este cálculo se utilizó la fórmula propuesta por la FAO (1996), adaptada por Ruiz, Savé, \& Herrera (2013):

$$
\text { (TDA): } \operatorname{Tas} a=\left[\frac{s_{2}}{s_{1}}\right]^{\frac{1}{n}}-1
$$

donde: TDA: tasa de cambio anual; $\mathrm{S}_{2}=$ superficie fecha $2 ; \mathrm{S}_{1}=$ superficie en la fecha $1 ; \mathrm{n}=$ es el número de años entre las dos fechas.

Con estas tasas y a través del álgebra de mapas se creó la cartografía de direccionalidad que indica la transición de una cobertura inicial a otra posterior, tomando como base el año 1973 y el año final el 2017. La direccionalidad de cambio se define aquí como la tendencia transicional en cobertura de un año a otro para un espacio determinado. Cabe resaltar que se determinó una direccionalidad única solo entre estos dos puntos de tiempo. El método de direccionalidad se centró entonces en establecer las transiciones de cobertura mediante una matriz de creación propia basada en la asignación de un sistema de codificación para cada cobertura. Esta matriz se elaboró con la herramienta Combine del ArcGis 10.5.

\subsection{Análisis estadístico}

La Tabla 1 detalla los repositorios de datos socioeconómicos y climáticos utilizados para el análisis estadístico. Para cada una de las variables, se calculó la tasa de cambio antes descrita (Ecuación 1).

Para examinar la correlación entre las variables y el cambio de cobertura de la tierra, se corrió una correlación de Spearman (Ecuación 2) debido a que la distribución de los datos no era normal. Se eligieron aquellos coeficientes de correlación mayores a \pm 0.6 , y donde existía una alta significancia entre las variables correlacionadas $(p>0.1)$. Por tanto, la ecuación de correlación utilizada fue:

$$
\mathrm{r}_{\mathrm{s}}=1-\frac{6 \sum_{i=1}^{n} d_{i}^{2}}{n^{3}-n}
$$

donde: $r_{s}=$ coeficiente de correlación Spearman; $d=$ diferencia entre los rangos $(\mathrm{X}-\mathrm{Y}) ; \mathrm{n}=$ total de datos. 
Tabla 1. Repositorios de datos libres para descargar las variables.

\begin{tabular}{ll}
\hline Variable & Repositorio y enlace web \\
\hline Censos poblacionales & $\begin{array}{l}\text { Institut Haïtien de Statistique et d'Informatique (IHSI) } \\
\text { (http://www.ihsi.ht/produit_demo_soc.htm) }\end{array}$ \\
\hline Temperatura y lluvia & $\begin{array}{l}\text { National Oceanic and Atmospheric Administration (NOAA) } \\
\text { (https://www.esrl.noaa.gov/psd/repository/entry/show?entryid=e570c8f9-ec09-4e89- } \\
\text { 93b4-babd5651e7a9) }\end{array}$ \\
\hline Índice de Desarrollo & $\begin{array}{l}\text { United Nations Development Programme (UNDP) } \\
\text { (http://hdr.undp.org/es/countries/profiles/HTI) }\end{array}$ \\
\hline Pumano & $\begin{array}{l}\text { Comisión Económica para América Latina y Publicaciones Estadísticas (CEPAL) } \\
\text { (http://interwp.cepal.org/sisgen/ConsultaIntegrada.asp?IdAplicacion=6yidTema=131yi } \\
\text { dIndicador=2204yidioma=e) }\end{array}$ \\
\hline
\end{tabular}

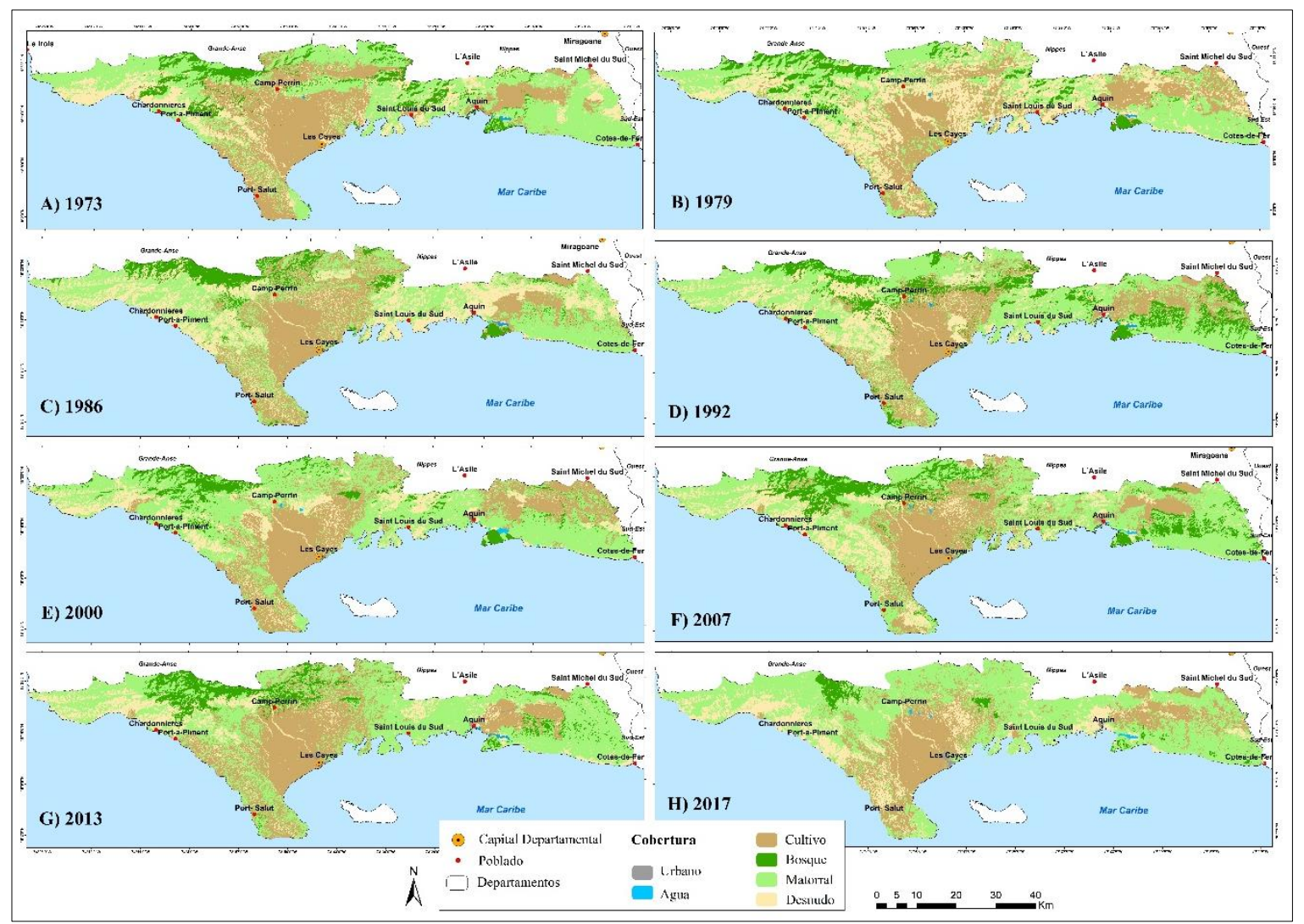

Figura 2. Serie de tiempo de la cobertura de la tierra en el Departamento Sur de Haití, de 1973 al 2017.

También se realizó una regresión linear múltiple (RLM) (Ecuación 3) para examinar la influencia de las variables en los cambios de las diferentes coberturas. El análisis RLM se trabajó con el software SigmaPlot v.13, donde se permite relacionar las tasas de cambio de las variables con las tasas de cambio de coberturas determinadas de una manera más automatizada. La ecuación de la RLM se muestra a continuación:

$$
y=b_{0}+b_{1} x_{1}+b_{2} x_{2}+b_{3} x_{3}+\ldots+b_{n} x_{n}+\varepsilon_{i}
$$

donde: $\mathrm{y}=$ variable dependiente (tasa de cambio de cobertura); $\mathrm{x}_{1}, \mathrm{x}_{2}, \mathrm{x}_{3} \ldots \mathrm{x}_{\mathrm{n}}=$ variables independientes; $\mathrm{b}_{0}$, $b_{1}, b_{2}, \ldots b_{n}=$ coeficientes de regresión.

Es importante recalcar que por ser los datos noparamétricos se usó la correlación de Spearman, sin embargo, se revisó la normalidad de las series para trabajar con RLM y al ser datos anuales no se hallaron mayores inconvenientes en la ejecución de las fórmulas.

\section{RESULTADOS}

\subsection{Dinámicas de cambio de cobertura de la tierra en el DS}

El análisis de los mapas de cobertura de la tierra (Fig. 2) determinó que, en el DS, y para el período estudiado, han predominado las coberturas de matorral, cultivos, y suelo desnudo respectivamente. Se evidenciaron zonas que desde el año 1973 hasta el 2017 se mantuvo la cobertura, tales son los casos de las áreas de matorrales entre Aquin y Côtes-de-Fer en el sector sureste del DS y cerca de las colindancias con Grand Anse, en el noroeste del DS; el gran reservorio agrícola en la llanura Les Cayes y el valle en Lalane, al noreste de Aquin; y la pequeña mancha boscosa del Parque Nacional Pic Macaya.

La cobertura boscosa, por su parte, tuvo una tendencia a la disminución, mas no así la cobertura impermeable asociada a usos urbanos. Los cuerpos de agua se expandieron y disminuyeron en extensión, sin embargo, esta cobertura no se tomó en cuenta para las correlaciones 
estadísticas ya que estas variaciones podrían depender de condiciones hidro-climáticas y geológicas propias de la región que no forman parte de esta investigación.

El área forestal mostró un promedio de área muy bajo entre los años 1973 y 2017, respecto a las anteriores coberturas, pero no superado por el urbano y cuerpos de agua, con los promedios de área mínimos dentro del DS. El promedio espacial en cuarenta y cuatro años fue dominado por la cobertura de matorral en todo el DS (45\%), que mostró un comportamiento parcialmente estable en comparación a las áreas dedicadas a cultivos, con área promedio del $27 \%$ y con una dinámica fluctuante en su extensión espacial, así como, con el suelo desnudo y su oscilante extensión, con un área promedio del $20 \%$. Es de resaltar que, la cobertura impermeable asociada a usos urbanos (Fig. 3A) mostró un evidente aumento del área a través del tiempo, sin embargo, denotó ser una cobertura mínima en extensión relativa al total de área del DS y con área promedio muy baja $(0.2 \%)$.
Para el año 1986, sin embargo, se observa una disminución de la cobertura urbana (Fig. 3A) de $1.62 \mathrm{~km}^{2}$, con una tasa de cambio de $-7.57 \%$ anual, asociado a los daños infraestructurales causados por el Huracán Allen en el año 1980 (EcuRed, 2015; Haïti-Référence, 2016). Luego, hubo una tendencia de crecimiento de la mancha urbana desde el año 1990 hasta el 2017, pero a ritmos variables. Entre el 2007 y el 2013, por ejemplo, hubo una tasa de cambio de $0.16 \%$ anual para esta cobertura, es decir, creció solo $0.07 \mathrm{~km}^{2}$, lo que en parte se explica por los efectos del terremoto del año 2010.

La cobertura de cultivo mostró una dinámica muy fluctuante (Fig. 3B). Hubo cuatro eventos en particular a nivel país que parecen haber incidido en la variación de área en esta cobertura: una gran sequía en 1986 que propició una disminución, un periodo de estabilidad económica hacia finales de los noventas aplicada por el UNSMIH (United Nations Support Mission in Haiti) que aumentó la extensión, un golpe de estado a inicios de los

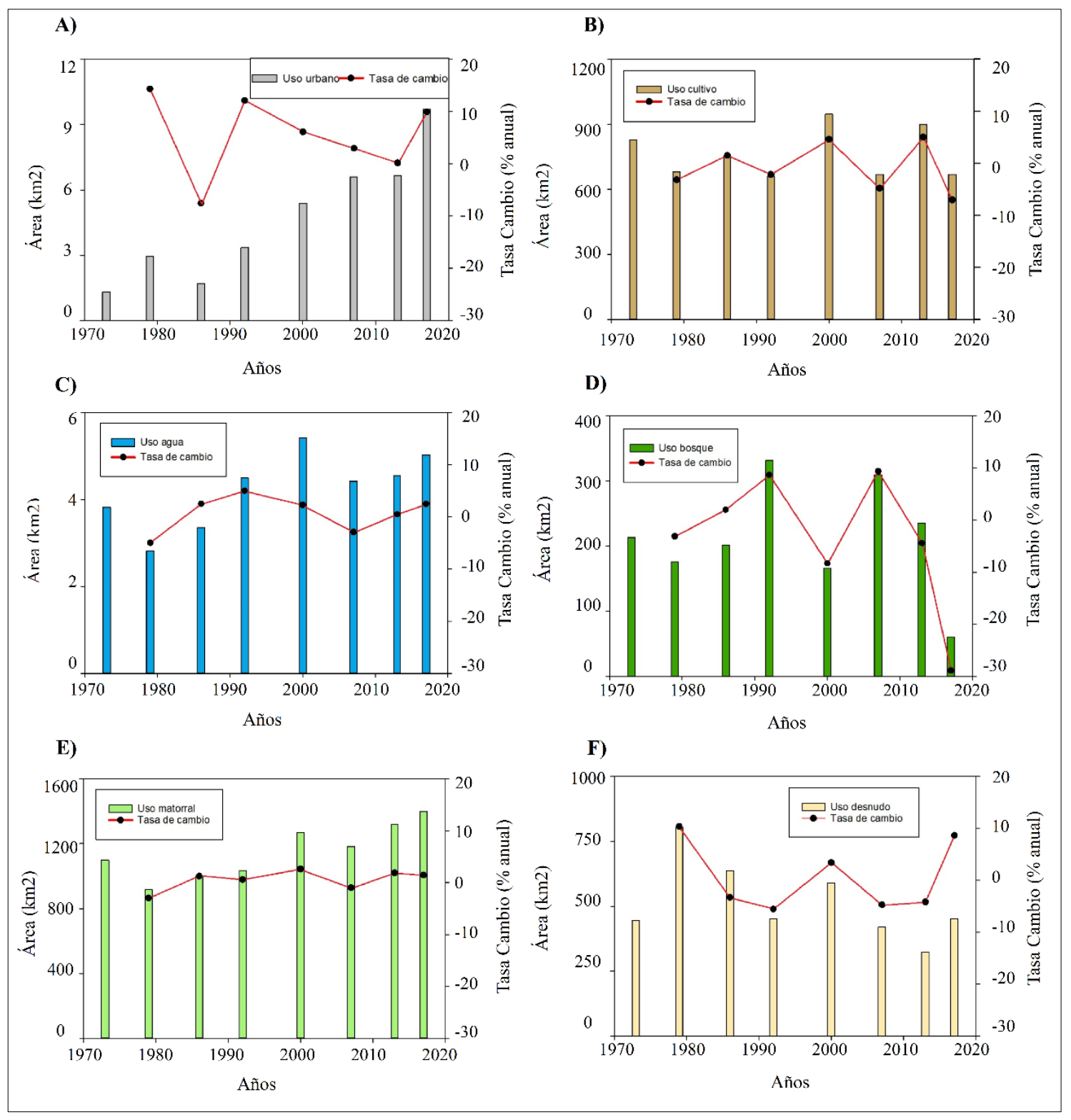

Figura 3. Gráficos de las áreas y tasas de cambio por cobertura por año. 
2000 que propició una disminución, y finalmente el huracán Matthew en el año 2016 que también afectó considerablemente las zonas de cultivos.

Los cuerpos de agua, como era de esperarse, presentaron fluctuaciones interanuales a raíz de la dinámica climática propia de la región, las condiciones física y químicas del agua y la estacionalidad en las imágenes satelitales utilizadas (temporadas de huracanes, época de lluvias convectivas, ENOS, época seca, etc.); sin embargo, en general muestran un aumento en todo el período de estudio (Fig. 3C). La cobertura boscosa tiende hacia la disminución en el período 1973-2017, mostrando también un comportamiento irregular en su patrón de cambio. Para el caso de esta cobertura, resaltan dos escenarios: un primero, entre los años 1992 y 2000, donde hubo procesos de deforestación importante vinculados a crisis económica y política entre 1991 y 1994 y el huracán Gordon en 1994 y cuyas consecuencias perduraron hasta años después; un segundo escenario, entre los años 2007 y 2017, que involucró una serie de eventos naturales que llevaron a otra gran ola de deforestación en la zona. Entre los eventos naturales destacan los huracanes Gustav (2008), Ike (2008), Sandy (2012) y Matthew (2016), así como el terremoto del año 2010. La cobertura boscosa entre los años 2013 y 2017, por ejemplo, disminuyó a un ritmo promedio de un $29 \%$ anual.

La cobertura de matorral muestra una dinámica de cambio más estable respecto a los demás (Fig. 3E). Sin embargo, entre 1973 y el 2017, sostuvo un aumento de $0.6 \%$ anual (un total de $300 \mathrm{~km}^{2}$ para el período). Como ya se mencionó, para el año 2017 el matorral mostró ser la cobertura dominante en todo el DS comprendiendo el $44 \%$ del total del área. Por otra parte, el suelo desnudo exhibe amplias variaciones en el período estudiado (Fig. 3F), con posibles influencias como la crisis política y económica de la primera mitad de los noventas reflejada en un aumento anual del 3.4\% entre los años 1992 y 2000 . También se registra un aumento importante en área de esta cobertura entre los años 2013 y 2017 (a un ritmo de 9\% anual) a raíz de la deforestación ocasionada por eventos naturales ya mencionados anteriormente.

\subsection{Dinámicas en la direccionalidad del cambio}

Para analizar las dinámicas de direccionalidad del cambio en las coberturas, se utilizaron una serie de mapas con base en una matriz codificada según cobertura. Con estos mapas, se pudo determinar mejor dónde una cobertura ganó y dónde perdió terreno. La Figura 4, por ejemplo, muestra las dinámicas de cambio espaciotemporal de la cobertura boscosa desde 1973 hasta el año 2017, particularmente un balance orientado hacia la pérdida forestal. El bosque presentó direccionalidades de cambio hacia todas las otras coberturas acá analizadas; es decir, las áreas con pérdida de bosque luego transaccionaron a coberturas de matorral, suelo desnudo, urbano, pastos y cuerpos de agua.

La Figura 5 muestra las áreas que fueron sometidas a un cambio de cobertura, así como las que no experimentaron cambios durante cuarenta y cuatro años, independientemente de la direccionalidad. Es de destacar que las áreas que no presentaron cambios históricos de cobertura son asociadas, en su mayoría, a áreas agrícolas, donde es posible que no hayan confluido factores naturales y sociales que motivasen hacia un cambio de cobertura.

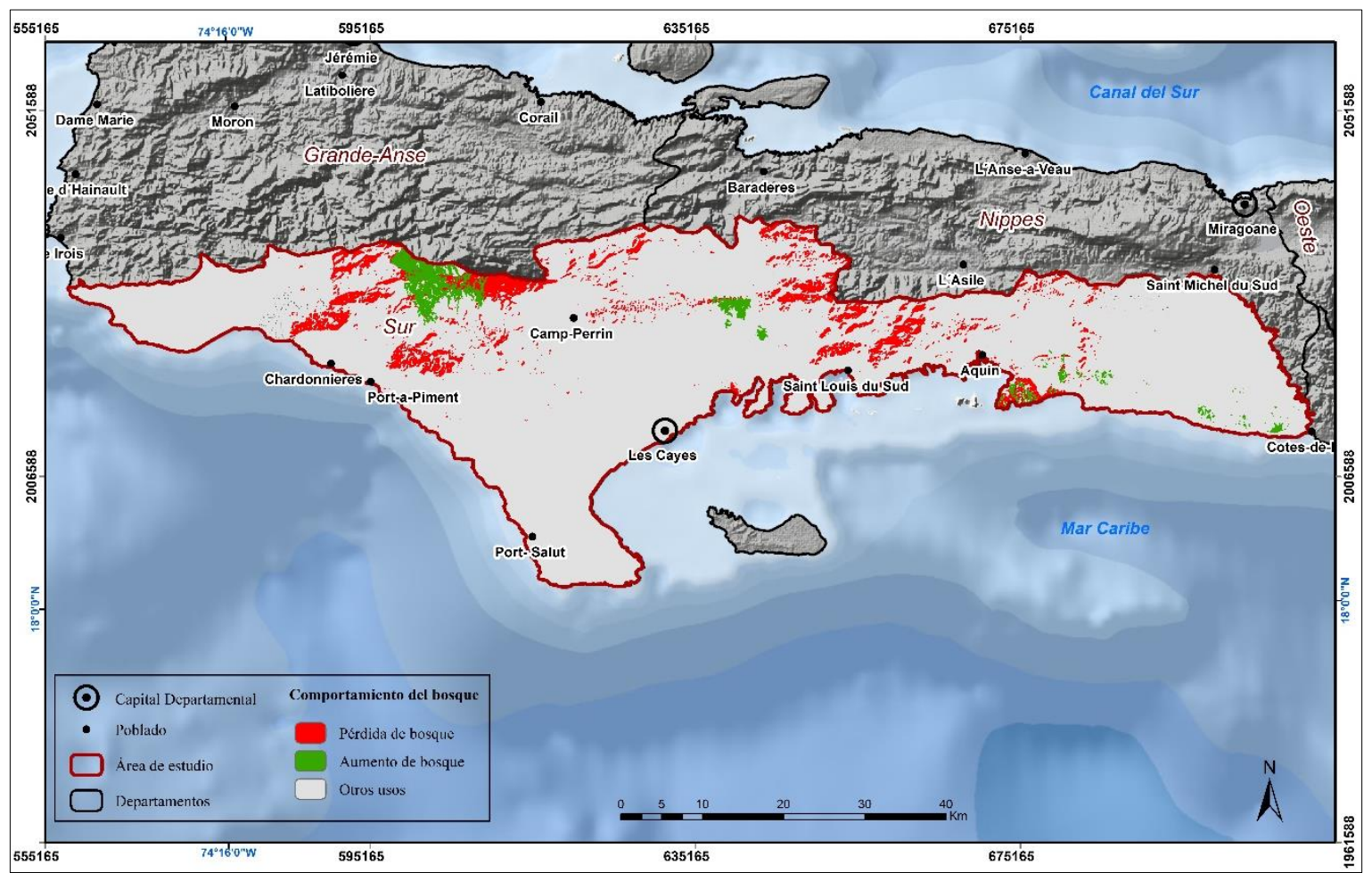

Figura 4. Comportamiento espacial de la cobertura de bosque, del año 1973 al año 2017. 


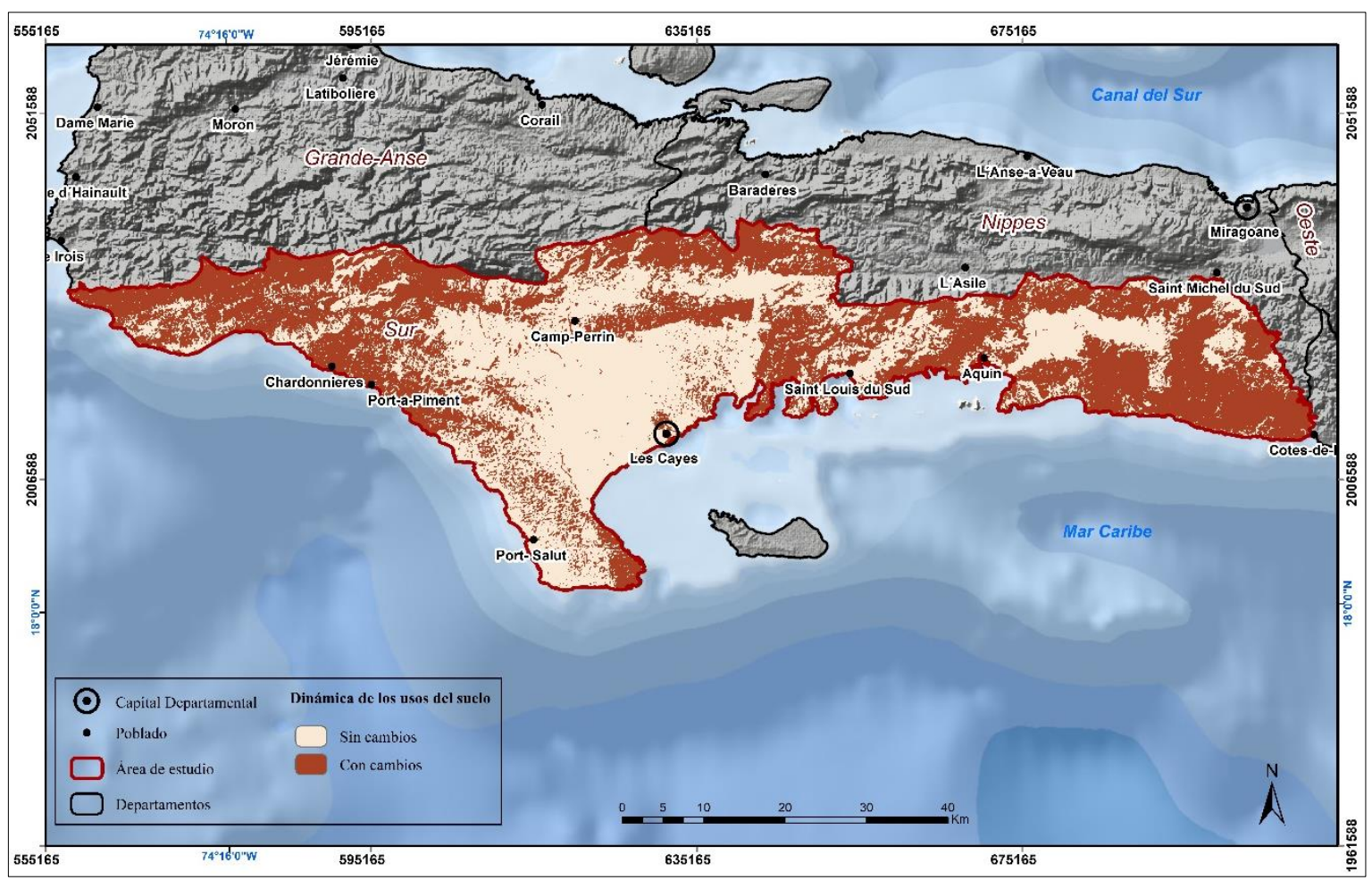

Figura 5. Estabilidad o cambio de la cobertura de la tierra, del año 1973 al año 2017.

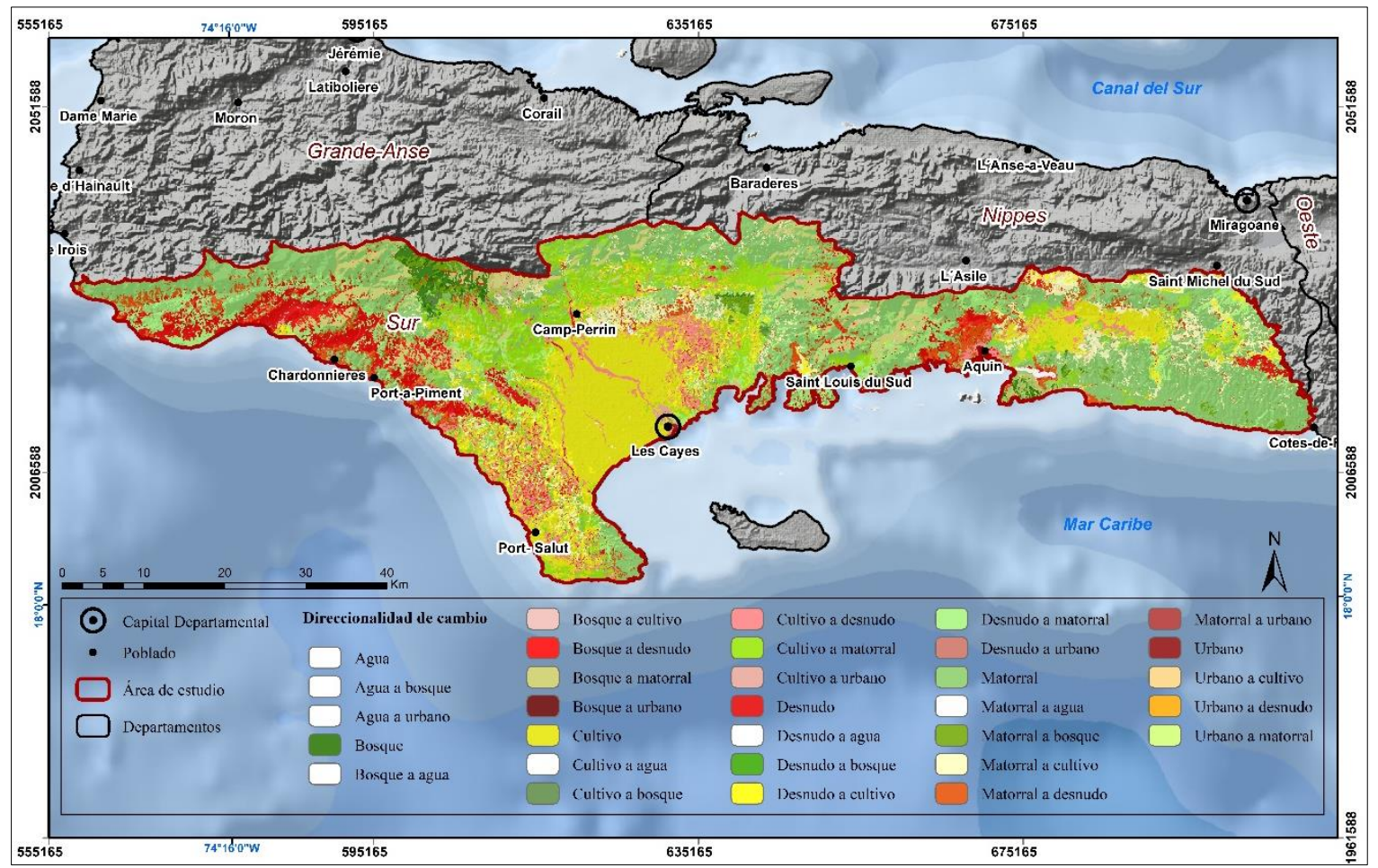

Figura 6. Direccionalidad de cambio de la cobertura de la tierra, 1973-2017. Departamento Sur de Haití

La Figura 6 detalla la direccionalidad de cambio entre las coberturas para el período 1973-2017. En tonalidades verdes se representan aquellos cambios en direccionalidad donde se ganó algún tipo de vegetación (de una cobertura sin vegetación hacia bosque o matorral), mientras que en tonos amarillos se representa la direccionalidad hacia un uso de cultivo y en tonos rojos hacia una pérdida de vegetación (de bosque o matorral hacia otras coberturas). El 23\% del Departamento Sur $\left(615 \mathrm{~km}^{2}\right)$ es más vulnerable a la degradación ambiental, ya que en el año 1973 presentaba alguna cobertura con vegetación (bosque, matorral o cultivo), y para el año 2017 cambiaron a áreas cubiertas de suelo desnudo, infraestructura urbana y residencial o cultivos rudimentarios 


\subsection{Análisis estadístico}

Resultó una correlación positiva entre la cobertura urbana, el matorral y la población, con una significancia de $p=0.01$, la cobertura de cultivo mostró una alta correlación con la tasa de cambio en la precipitación, con $p=0.05$. De la misma forma, se identificó una correlación significativa entre el cambio del suelo desnudo y la tasa de cambio de población con $p=0.08$, y la cobertura de matorral mostró una alta correlación con tres variables: tasa de cambio de la población, con $p=0.003$, tasa de cambio de la temperatura y el IDH ( $p=0.04$ para ambos). Además, no se encontró correlación significativa entre los cambios de cobertura de bosque e infraestructura/urbano con las variables consideradas; es decir, ninguna de las variables parece influir en el cambio de dichas coberturas para el período estudiado. Las demás variables no alcanzaron un nivel de correlación mínimo. Se trató de un ejercicio para encontrar relaciones entre variables, lo que no representa una causa al cambio de alguna cobertura.

Tabla 2. Correlaciones significativas de variables seleccionadas.

\begin{tabular}{lccc}
\hline Cobertura & Variable & $\mathrm{R}$ & $p$ \\
\hline Urbanos & Población & 0.86 & 0.01 \\
\hline Cultivo & T.C precipitación & 0.71 & 0.05 \\
\hline \multirow{3}{*}{ Matorral } & T.C población & 0.87 & 0.003 \\
& T.C temperatura & 0.95 & 0.04 \\
& T.C. IDH & 0.98 & 0.04 \\
\hline Desnudo & T.C población & 0.66 & 0.08 \\
\hline
\end{tabular}

\section{DISCUSIÓN}

\subsection{La utilidad de sensores remotos para detectar cambios de cobertura de la tierra en territorios con recursos limitados}

Una herramienta que indudablemente ha dado un empuje significativo al estudio del cambio global es la teledetección desde satélites orbitales (Duarte et al., 2006; Chuvieco, 2002). Uno de los principales usos de estos sensores remotos en el área ambiental ha sido el monitoreo de la cobertura de la tierra y la cuantificación de su cambio (Banskota et al., 2014; Chuvieco, 2002). Por ejemplo, los instrumentos de observación de la Tierra han sido ampliamente utilizados en la evaluación y el seguimiento de la dinámica forestal en el espacio y en el tiempo de manera continua. Además, dada la compleja dinámica espacial y temporal del cambio global, las series temporales de observación son indispensables en investigaciones sobre este tema, pues permiten estudiar patrones de cambio.

Estas funciones de los instrumentos de teledetección son especialmente importantes para procesos de planificación territorial y toma de decisiones en general en sitios difíciles de acceder o con recursos limitados para el monitoreo constante de los cambios en el paisaje. Bajo tales fundamentos, la presente investigación replicó metodologías de diversos estudios en donde el objetivo era clasificar imágenes (Ayala \& Menenti, 2001; Chuvieco, 2002; Martínez, 2005; Nazareno, 2014; Sacristán, 2006; Tovar, 2013) y cuantificar el cambio de cobertura de la tierra (Falcón, 2014; Lambin, Geist, \& Lepers, 2003; López, Balderas, Chávez, Juan, \& Gutiérrez, 2014; Mas et al., 2004; Mas, Velázquez, \& Couturier, 2009; PonceOpazo, 2010; Rindfuss et al., 2015; Ruiz et al., 2013), pero aplicado a territorios donde la calidad y cantidad de información espacial es limitada, como es el caso de Haití.

Los datos geoespaciales en países en vías de desarrollo son escasos, incompletos, o de acceso restringido. El uso de sensores remotos y de productos espaciales de libre acceso (como los utilizados en esta investigación) generan ventajas y oportunidades de desarrollo en todos los sectores estatales, ya que ayudan a proveer información útil y precisa para tomar decisiones acertadas. Sin embargo, el uso de tecnología espacial es un desafío importante para los entes estatales y privados en países con recursos limitados, al no contar con el personal adecuado y la infraestructura tecnológica básica para tratar dicha información (Di Martino, Iodice, Pansera, Riccio, \& Ruello, 2007), lo que investigaciones como la nuestra, pone a disposición los datos para acceso abierto. Los datos de sensores remotos ofrecen una amplia cobertura espacial de información multitemporal, vitales para el manejo, asesoría y monitoreo de recursos naturales, además que permite el seguimiento y estudio en desastres socioambientales, cambio climático y degradación ambiental, entre otros (Pramanik, 1993). Por lo tanto, reconocemos las limitaciones relacionadas a la cantidad de variables sociales y biofísicas utilizadas en esta investigación, en gran medida debido a la falta de disponibilidad de datos confiables para Haití.

\subsection{La relación de cambios de cobertura de la tierra con otros factores sociales y naturales para una caracterización del cambio global}

Esta investigación examinó la relación entre ciertas variables sociales y biofísicas con el cambio de cobertura de la tierra en el DS de Haití. En efecto, el estudio del cambio global implica considerar las interrelaciones complejas entre factores sociales y biofísicos y las manifestaciones en el espacio y tiempo de estas interrelaciones. También conlleva el considerar cómo los eventos socioambientales a escalas subglobales inciden de forma directa o indirecta en los patrones de los sistemas terrestres a nivel global. En esta investigación, se examinaron las interacciones entre componentes ambientales y socioeconómicas para el DS de Haití, como parte de una dinámica global donde los cambios a escalas más finas son acumulativos y llegan a incidir en el funcionamiento de sistemas de soporte de vida a escalas mayores, incluida la global.

Consideramos importante contextualizar los resultados obtenidos en esta investigación con el fin de situarlos mejor entre las dinámicas de cambio a nivel regional y mundial. La deforestación, es clave a la hora de comparar casos. En Europa, por ejemplo, cerca de 8.8 millones de hectáreas de bosque natural en Europa fueron deforestadas entre 2010-2015 (Fern, 2017), un área similar a Suecia. En África se han perdido 5,264,000 ha (51\% del continente) de la cobertura forestal, mientras que en Asia aproximadamente 454,000 ha (4\% del continente) y en Latinoamérica alrededor de 4,588,000 ha $(45 \%$ de la región) entre los años 1990 y 2000 (Geist \& Lambin, 2001). En los últimos 300 años de existencia humana, se estima que se ha perdido entre un 15 y $30 \%$ de la cobertura forestal por actividades agropecuarias (Klein-Goldewijk 
\& Ramankutty, 2010; Turner, 1988). Entretanto, la tasa de cambio en la cobertura forestal en la región tropical del Planeta del año 1990 al 2010 fue de -0.36 en promedio (D'Annunzio, Lindquist, \& MacDicken, 2017). La deforestación sigue siendo una apremiante problemática ambiental en muchas regiones del Planeta, en especial en zonas tropicales, con causas directas como la expansión de la mancha urbana, expansión agrícola y ganadera, y extracción maderera, así como causas indirectas como demandas de mercado, dinámicas migratorias, y políticas gubernamentales (Geist \& Lambin, 2001; Taubert et al., 2018; Watson et al., 2000). De hecho, entre los años 2000 y 2005, la deforestación tropical se correlacionó positivamente con el aumento de la población urbana y las exportaciones de productos agrícolas (DeFries, Rudel, Uriarte, \& Hansen, 2010).

Para el caso específico de Haití, y según datos de PNUMA (2013), existe entre un 2 y $4 \%$ de bosque en la actualidad. Sin embargo, Churches, Wampler, Sun, \& Smith (2014) confirman que, para todo el país, existe un $29.4 \%$ de cobertura boscosa, basado en sensado remoto de alta definición. En el DS en particular, en el año 2017, presentó un $2.3 \%$ de cobertura forestal, según datos de nuestra investigación. Tomando como base a los resultados de Churches et al. (2014), el DS cuenta con el $8.16 \%$ del bosque en Haití. Jeune, de Souza, Rocha Franceline, Fernandes Filho, \& Crusoé Rocha (2018) revelaron que, en la región oeste de Haití, la tasa de cambio del bosque fue de $-3.4 \%$ entre los años 2000 y 2014 , y en el DS, datos de nuestra investigación reportó un leve crecimiento de $2.5 \%$ en la tasa de cambio del bosque.

En la cuenca Fonvèrèt, al suroeste de Haití, la deforestación fue mayormente propiciada por la instauración de SHADA (The Société HaitianeAméricaine de Développement Agricole) para sembrar caucho desde 1941 (Versluis \& Rogan, 2009), y en el DS la deforestación la consideramos una problemática poscolonial. La transformación de coberturas boscosas en el Pic Macaya, que pasaron a ser suelo desnudo y tierras agrícolas, fueron causadas por la colonización agraria en búsqueda de suelos para siembra de hortalizas y acciones políticas desde antes de 1987 (Vital, 2008), hecho que en la presente investigación se constata puntualmente con la direccionalidad de cambio (Fig. 6). Esto hace indicar que, en Haití la dinámica de la cobertura forestal no es homogénea, y los procesos de deforestación están en función de factores biofísicos y sociales propios de cierta región.

La tasa de cambio de la precipitación condujo a un posible aumento de la tasa de cambio de las áreas con cultivos. Por otro lado, la tasa de cambio de la población propició un aumento en la tasa de cambio del suelo desnudo, lo que hace pensar en el efecto que ejerció el crecimiento poblacional sobre los recursos del DS, modificando la cobertura de la tierra. La variabilidad climática y la dinámica poblacional son factores que ejercen cambios directos en la cobertura de la tierra (Zondag \& Borsboom, 2009; Lambin et al., 2001).

Los resultados aquí presentados también ayudan a identificar patrones espaciotemporales en la deforestación en el DS. Por ejemplo, los mapas evidencian los pocos remanentes de áreas forestales, así como una trayectoria de deforestación que se explica por acontecimientos sociohistóricos de larga data desde épocas coloniales (Agencia Suiza para Desarrollo y Cooperación, 2016; González \& Manero, 2011; PNUMA, 2013; Vitale, 1987).
Es decir, en Haití la deforestación a mediana y gran escala data de la época colonial y, en la actualidad, se continúa esa tendencia hacia la pérdida de cobertura forestal.

Sin embargo, la determinación de las causas de los procesos de deforestación trascendía el alcance de esta investigación, y ante los pocos de estudios que modelen esta causalidad en Haití, son necesarias investigaciones en esta línea. Se estima que esta problemática está estrechamente ligada al uso de madera como fuente de energía en hogares de escasos recursos, como producto comercial de venta hacia lo interno y al exterior de Haití, así como también para instaurar espacios agrícolas rudimentarios y crear asentamientos poblacionales informales (González \& Manero, 2011). No obstante, a pesar de contar con una limitante de datos espaciales para el país, se lograron identificar los espacios alterados ambientalmente, lo que sienta una base para fundamentar leyes y reglamentos en pro del manejo de los recursos naturales de Haití.

\section{CONCLUSIONES}

En esta investigación se logró expresar la dinámica espaciotemporal del cambio de cobertura de la tierra en el DS, y mediante la cartografía de direccionalidad de cambio, se evidenciaron las zonas más vulnerables ante el cambio de cobertura boscosa, donde los efectos por la erosión del suelo, la escorrentía superficial y la degradación del suelo tienden a ser más prominentes. La distribución de estas zonas es espacialmente focalizada, es decir, no se puede concluir que todo el DS expone un daño ambiental considerable por el cambio de cobertura sin planificación. Sin embargo, más del $50 \%$ del DS (incluyendo las partes altas) presenta coberturas vegetativas (matorral y parches de bosque) que ayudan a la retención del suelo e infiltración del agua, pero menos del $10 \%$ del departamento muestra una recuperación de áreas boscosas. Esto es en parte facilitado por un déficit de políticas ambientales de inversión económica estatal, aunado a la poca conciencia ambiental de la población. Esto ayuda a concluir, con base a los resultados, que los factores socioeconómicos fueron los más influyentes en el cambio de cobertura de la tierra, y, por tanto, a la vulnerabilidad ambiental del DS.

Por último, esta investigación contribuye a la comprensión de cómo influyeron los eventos naturales, políticos, económicos y sociales en el cambio de cobertura de la tierra en el DS de Haití, siendo esto útil para los tomadores de decisiones a diversos niveles de gobernanza, particularmente aquellos encargados de liderar un desarrollo social y económico a través de la panificación territorial y un manejo sostenible de los recursos naturales.

\section{AGRADECIMIENTOS}

Al proyecto WaterClima-LAC, Gestion de Zonas Costeras, a través del cofinanciamiento de la Unión Europea, por el apoyo financiero. Al M.Sc. William Watler de CATIE, por su guía y ayuda en campo. Los autores agradecen el apoyo parcial del Observatorio del 
Agua y Cambio Global (OACG) de la Escuela de Geografia, Universidad de Costa Rica.

\section{REFERENCIAS}

Administración Nacional Oceánica y Atmosférica, NOAA. (2017). Climate data repository. U.S. Department of Commerce. https://www.noaa.gov/

Agencia Suiza para Desarrollo y Cooperación, CONSUDE. (2016). Preservación del bosque de los pinos en Haití: Haití: Salvar los árboles - mejorar las vidas. Latin Brief, 2, 4 pp. Disponible en:

https://www.eda.admin.ch/dam/deza/es/documents/pub likationen/briefing-papers/latin-brief-haiti160615_ES.pdf

Ayala, R. M., \& Menenti, M. (2001). Metodología para la búsqueda del mejor clasificador de imágenes de satélite. Teledetección, Medio Ambiente y Cambio Global, 469-472. Disponible en: http://www.aet.org.es/congresos/ix/Lleida103.pdf

Banco Mundial. (2017). Haití. Disponible en: http://datos.bancomundial.org/pais/haiti

Banskota, A., Kayasthan, N., Falkowski, M. J., Wulder, M. A., Froese, R. E., \& White, J. C. (2014). Forest monitoring using Landsat time series data: A review. Canadian Journal of Remote Sensing, 40(5), 362-384. doi.org/10.1080/07038992.2014.987376

Barthelemy, M., \& Barthelemy, C. (2003). Haïti, la perle nue. Vents d'ailleurs, France.

Carr, D. L. (2004). Factores demográficos proximales y deforestación en las fronteras agrícolas tropicales. Population and Environment, 25(6), 1-41.

Chávez, P. S. (1988). An improved dark-object subtraction technique for atmospheric scattering correction of multispectral data. Remote Sensing of Environment, 24(3), 459-479. https://doi.org/10.1016/0034-4257(88)90019-3

Churches, C. E., Wampler, P. J., Sun, W., \& Smith, A. J. (2014). Evaluation of forest cover estimates for Haiti using supervisedclassification of Landsat data. International Journal of Applied Earth Observation and Geoinformation, 30, 203-216. https://doi.org/10.1016/j.jag.2014.01.020

Chuvieco, E. (2002). Teledetección Ambiental. Barcelona, España: Editorial Ariel.

D’Annunzio, R., Lindquist, E. \& MacDicken, K. (2017). Global forest land-use change from 1990 to 2010: An update to a global remote sensing survey of forests. Forest Resources Assessment Working Paper, 187, 114. Disponible en: http://www.fao.org/3/a-i5098e.pdf

DeFries, R. S., Rudel, T., Uriarte, M., \& Hansen, M. (2010). Deforestation driven by urban population growth and agricultural trade in the twenty-first century. Nature Geoscience, 3, 178-181. doi:10.1038/NGEO756

Departamento de Asuntos Económicos y Sociales, DAES. (2017). World Population Prospects: The 2017 Revision. Organización de Naciones Unidas. Nueva York. Disponible en: https://population.un.org/wpp/Publications/Files/WPP2 017_KeyFindings.pdf
Di Martino, G., Iodice, A., Pansera, M., Riccio, D., \& Ruello, G. (2007). Remote sensing for developing countries: Landsat data and Gis. Rivista Italiana di Telerilevamento, 38, 3-13. Disponible en: http://wpage.unina.it/gerardo.dimartino/papers/DiMarti no.pdf

Duarte, C. M. (coord.) et al. (2006). Cambio global: Impacto de la actividad humana sobre el sistema Tierra. Consejo Superior de Investigaciones Científicas. Madrid, España. Disponible en: http://digital.csic.es/bitstream/10261/27310/1/cambioG lobal.pdf, $170 \mathrm{pp}$.

EcuRed. (2015). Desastres naturales en Haití. Disponible en: https://www.ecured.cu/ Desastres_naturales_en_Hait\%C3\%AD.

Equihua Zamora, M., Hernández Huerta, A., Pérez Maqueo, O., Benítez Badillo, G., \& Ibañez Bernal, S. (2015). Cambio global: el Antropoceno. Ciencia Ergosum, 23(1), 67-75. Disponible en: https:// dialnet.unirioja.es/descarga/articulo/5379210.pdf

Falcón, O. (2014). Dinámica de cambio de uso/cobertura del suelo, en una región del Estado de Quintana Roo, México: El impacto de las políticas gubernamentales sobre el Manejo Forestal Comunitario (Tesis de grado). Universidad Autónoma de México. Disponible en: http:// 132.248.9.195/ptd2014/enero/0707773/0707773.pdf

Fern. (2017). Tackling deforestation and forest degradation: a case for EU action in 2017. Disponible en: https://www.forestpeoples.org/ sites/default/files/documents/deforestation-4.pdf

Geist, H. J., \& Lambin, E. F. (2001). What drives tropical deforestation? A meta-analysis of proximate and underlying causes of deforestation based on subnational case study evidence. LUCC Report Series 4, 136 pp. CIACO, Universidad de Louvain, Louvainla-Neuve, Bélgica. Disponible en: https://www.pikpotsdam.de/members/cramer/ teaching/0607/Geist_2001_LUCC_Report.pdf

González, M., \& Manero, A. (2011). El conflicto de Haití. Conflictos Internacionales Contemporáneos. 291 pp. https://publicaciones.defensa.gob.es/el-conflictode-haiti.html

Haïti-Référence. (2016). Catastrophes Naturels: Cyclones y Ouragans. Disponible en: https://www.haiti-reference.com/pages/plan/ geographie-et-tourisme/milieu-naturel/desastres-etaccidents/cyclones-et-ouragans/

Houghton, R. (1994). The worldwide extent of land-use change. BioScience, 4(5), 305-313. https://doi.org/10.2307/1312380

Jeune, W., de Souza, E., Rocha Franceline, M., Fernandes Filho, E. I., \& Crusoé Rocha, G. (2018). Land use and land cover dynamics in western Haiti. In: 21WCSS: Proceedings of the 21st World Congress of Soil Science, 2018, August 12-17; Rio de Janeiro, Brazl: SBCS, Vol. II, p. 393. Disponible en: http://www.21wcss.org/docs/ Proceedings_of_the_21WCSS_Volume_II.pdf

Klein-Goldewijk, K., \& Ramankutty, N (2010). Land use changes during the past 300 years. Land use, land cover and soil sciences, 1, $22 \mathrm{pp}$. Disponible en: https://www.researchgate.net/publication/ 
242217587_Land_use_changes_during_the_past_300_ years

Lambin, E. F., Turner, B. L., Geist, H. J., Agbola, S. B., Angelsen, A., ..., et al. (2001). The causes of land-use and land-cover change: moving beyond the myths. Global Environmental Change, 11(4), 261-269. https://doi.org/10.1016/S0959-3780(01)00007-3

Lambin, E., \& Geist, H. (Eds.) (2006). Land-use and land-cover change: Local processes and global impacts. Springer, Alemania.

Lambin, E., \& Meyfroidt, P. (2011). Global land use change, economic globalization, and the looming land scarcity. PNAS, 8(9) 3465-3472. https://doi.org/10.1073/pnas.1100480108

Lambin, E., Geist, H., \& Lepers, E. (2003). Dynamics of land use and land cover change in tropical regions. Annual Review of Environment y Resources, 28, 205245. doi:10.1146/annurev.energy.28.050302.105459

López, V. H., Balderas, M. A., Chávez, M. C., Juan, J. I., \& Gutiérrez, J. G. (2014). Cambio de uso de suelo e implicaciones socioeconómicas en un área mazahua del altiplano mexicano. CIENCIA ergo-sum, 22(2) 136144. Disponible en: https://dialnet.unirioja.es/ descarga/articulo/5116566.pdf

Martínez, M. (2005). Percepción Remota: Fundamentos de Teledetección espacial. México: Comisión Nacional de Agua Subdirección General de Programación, Subgerencia de Informática y Sistema Geográfico del Agua. Jefatura de Control Cartográfico. Disponible en: http://siga.cna.gob.mx/

SIGA/Percepcion/Fundamentos $\% 20$ de $\% 20$ teledetecci $\% \mathrm{C} 3 \% \mathrm{~B} 3 \mathrm{n} \% 20$ espacial.PDF

Mas, J-F., Velázquez, A., Díaz-Gallegos, J. R., MayorgaSaucedo, R., Alcántara, C., ..., et al. (2004). Assessing land use/cover changes: a nationwide multidate spatial database for Mexico. International Journal of Applied Earth Observation and Geoinformation, 5(4), 249-261. https://doi.org/10.1016/j.jag.2004.06.002

Mas, J., Velázquez, A., \& Couturier, S. (2009), La evaluación de los cambios de cobertura/uso del suelo en la República Mexicana. Investigación Ambiental, 1(1), 23-39.

Meyer, W., \& Turner, B. (2002). The Earth transformed: trends, trajectories and patterns. En: Johnston R., Taylor, P., \& Watts, M. (Eds.). Geographies of global change: Remapping the world (2a ed.) (pp. 364-376). UK, Oxford: Blackwell.

Nazareno, M. (2014). Métodos de clasificación de imágenes satelitales para la determinación de usos del suelo agrícola en el Partido de Tandil (Provincia De Buenos Aires, Argentina). Universidad Nacional del Centro de la Provincia de Buenos Aires. Disponible en: http://www.formagro.com/ recursos/descargas/Metodos-de-clasificacion-digitalde-imagenes-satelitales-para-clasificacion-de-uso-desuelos.pdf

Ponce-Opazo, B. (2010). Detección y análisis del cambio de uso del suelo en la zona centro - norte de la provincia de Capitán Prat, XI Región período 1984 2003. Tesis de grado, Facultad de Ciencias Forestales y Recursos Naturales, Universidad Austral de Chile, Valdivia, Chile. Disponible en: http://cybertesis.uach.cl/tesis/uach/2010/fifp792d/doc/f ifp792d.pdf
Pramanik, M. (1993). Satellite remote sensing and its application to the needs of developing countries: An Asian perspective. Selected Papers on Remote Sensing, Satellite Communications, and Space Science. Seminars of the United Nations Program on Space Applications, Vol. 4, 145-156. Disponible en: http://adsabs.harvard.edu/full/1993UNPSA...4..145P

Programa de las Naciones Unidas para el Ambiente. (2013). Haití-República Dominicana: Desafios ambientales en la zona fronteriza. Nairobi, Kenia: Programa de las Naciones Unidas para el Medio Ambiente. Disponible en: https://postconflict.unep.ch/publications/UNEP_HaitiDomRep_border_zone_SP.pdf

Rindfuss, R. R., Entwisle, B., Walsh, S. J., An, L., Badenoch, N., ..., et al. (2015). Land use change: complexity and comparisons. Journal of Land Use Science, 3(1), 1-10. https://doi.org/10.1080/17474230802047955

Rounsevell, M. (2006). Global environmental change. En: Geist, H. (Ed.), Our Earth's Changing Land: An Encyclopedia of Land-Use and Land-Cover Change (pp. 254-256). Estados Unidos: Greenwood Publishing Group.

Ruiz, V., Savé, R., \& Herrera, A. (2013). Análisis multitemporal del cambio de uso del suelo, en el Paisaje Terrestre Protegido Miraflor Moropotente Nicaragua, 1993-2011. Ecosistemas, 22(3), 117-123. doi: 10.7818/ECOS.2013.22-3.16

Sacristán, F. (2006). La teledetección satelital y los sistemas de protección ambiental. AquaTIC, 24, 13-41. Disponible en: http://www.revistaaquatic.com/aquatic/pdf/24_02.pdf

Taubert, F., Fischer, R., Groeneveld, J., Lehmann, S., Müller, M. S., ..., et al. (2018). Global patterns of tropical forest fragmentation. Nature, 554, 519-522. doi:10.1038/nature25508

Tovar, W. (2013). Propuesta de clasificación de cobertura/uso de la tierra en los Andes. Caso: cuenca del río Grita, Venezuela. Tesis de grado, Instituto de Ciencias Ambientales y Ecológicas (ICAE), Universidad de Los Andes, Venezuela. Disponible en: http://www.saber.ula.ve/bitstream/ 123456789/39866/1/Tovar2013.pdf

Turner, B. (1988). The earth as transformed by human action. The Professional Geographer, 40(3), 340-341. https://doi.org/10.1111/j.0033-0124.1988.00340.x

Veldkamp, A., \& Lambin, E. (2001). Predicting land-use change. Agriculture, Ecosystems and Environment, 85, 1-6. doi:10.1016/S0167-8809(01)00199-2

Versluis, A., \& Rogan, J. (2009). Mapping land-cover change in a Haitian watershed using a combined spectral mixture analysis and classification tree procedure. Geocarto International, 25, 1-19. doi:10.1080/10106040902977584

Vital, J. (2008). Land Use/Cover Change Using Remote Sensing and Geographic Information Systems: Pic Macaya National Park, Haiti. Master's Thesis, Michigan Technological University, 2008. https://digitalcommons.mtu.edu/etds/138 
Vitale, L. (1987). Haití: Primera nación independiente de América Latina. Revista EDM, 43. Disponible en: https://revistaedm.com/verNotaRevistaTeorica/516/hait i-primera-nacion-independiente-de-america-latina

WaterClima-LAC. (2017). Proyecto Piloto B. Aquin, Zona Costera Sur de Haití (Caribe). EuropeAid Project.

Watson, R. T., Noble, I. R., Bolin, B., Ravindranath, N. H., Verardo, D. J., \& Dokken, D. J. (Eds.) (2000). Land Use, Land Use Change and Forestry. Cambridge, England: Cambridge University Press. Pp. 875.

Disponible en: https://www.ipcc.ch/report/ land-useland-use-change-and-forestry/
Zondag, B., \& Borsboom, J. (2009). Driving forces of land-use change. 49th ERSA Conference, Lodz, Poland, 1-16. Disponible en: https://www.researchgate.net/publication/285854006_ Driving_forces_of_land-use_change 